Asymptomatic benign familial spinal muscular atrophy with hypertrophy of the calves and high creatine kinase levels

Sir: In spinal muscular atrophies, hypertrophy of the calves ${ }^{1-4}$ and an increase of serum creatine kinase (CK) activity 356 have been found. We report an asymptomatic 33 year old man, with only hypertrophy of the calves and high blood level of CK. Further investigations indicate that the patient had benign familial spinal muscular atrophy.

The proband was a 33 year old Japanese man. The family history was negative for neuromuscular disorders. At the age of 32 years, raised serum CK activity $(762 \mathrm{U} / \mathrm{l})$ (normal; 25-170) was noted incidentally. He visited our clinic at the age of 33 years because of the raised CK level. There was gross hypertrophy of the calves. No other general or neurological abnormalities were observed. Muscle strength and tendon reflexes were normal. Routine laboratory data including erythrocyte sedimentation rate, blood cell count, and serum chemistry were normal except for serum CK, GPT, $\mathrm{LDH}$, and aldolase. Serum CK activity ranged from 654 to $820 \mathrm{U} / 1$ (normal; 25-170), aldolase was 7.8-11.0 U/1 (normal; 1.2-7.6), GPT was $66 \mathrm{U} / 1$ (normal; 5-47), and LDH was 202-286 U/1 (normal; 125-220). Levels of GOT were within normal limits. Serum CK isozymes consisted of 0\% BB type, 3\% MB type, $91 \%$ MM type, and $5 \%$ albumin. There was abnormality in electrocardiogram. Serum levels of pyruvate and lactic acid were normal at rest and after exercise. Needle electromyogram (EMG) revealed reduced interference patterns and high amplitude, long duration potentials in the right deltoid, triceps brachii, extensor digitorum communis, and quadriceps femoris muscles. The high amplitude potentials were polyphasic and firing at high rates. No spontaneous activity was recorded in the EMG. Motor and sensory nerve conduction velocities were within normal limits in the upper and lower extremities. CT of the legs revealed true hypertrophy of the calf muscles. A muscle tissue was biopsied from the quadriceps femoris. The specimen was rapidly frozen in isopentane cooled in liquid nitrogen, and cryostat sections were stained by histological and histochemical methods of Dubowitz. ${ }^{7}$ The biopsy specimen included 840 muscle fibres. Average size of the muscle fibres was $77 \mu \mathrm{m}$. A few small angulated fibres (about $1 \%$ of all of the fibres) were scattered. Hypertrophic fibres, more than $80 \mu \mathrm{m}$ in diameter, were found in $41 \%$ of the total. There was no necrotic fibre or intersitial fibrosis. No ragged red fibres were found in modified Gomori trichrome stain. The standard ATPase reaction ( $\mathrm{pH}$ 9.4) demonstrated that all the fibres were uniformly type 2 . Acid pre-incubation $(\mathrm{pH}$ 4.6 and 4.3 ) of the ATPase reaction revealed that the muscle consisted of type $2 \mathrm{a}(38 \%)$ and $2 \mathrm{~b}$ fibres $(62 \%)$. Average diameter of type $2 \mathrm{a}$ fibres was $81 \mu \mathrm{m}$, and that type of $2 \mathrm{~b}$ fibres $74 \mu \mathrm{m}$. Fibre type grouping was found with clusters of both type $2 a$ and type $2 b$ fibres. The small fibres belonged to type $2 b$.

We examined the patient's father who was 61 years old. General and neurologic examinations were normal. There was no hypertrophy of the calves. The serum chemistry revealed elevated activities of CK (342 U/1), LDH (277 U/1), and aldolase (9.4 U/1). The needle EMG revealed high amplitude, long duration potentials, which were also polyphasic, in the right quadricep femoris and extensor digitorum communis. Polyphasic, long duration potentials were found in the right deltoid, biceps brachii, and triceps brachii. No spontaneous activity was recorded in the EMG.

The proband is characterised by hypertrophy of the calves, high $\mathrm{CK}$ level, and abnormalities in EMG and muscle biopsy. The findings of EMG and muscle pathology suggest impairment of the lower motor neuron, which implies that the patient is asymptomatically affected with spinal muscle atrophy. In the muscle pathology, the muscle fibres consisted of only type 2 with fibre type grouping including clusters of both types $2 a$ and $2 b$. Marked tendency of fibre type grouping might cause the appearance of uniform type 2 fibres in the small biopsy specimen. In the mild form of spinal muscle atrophy, large muscle fibres often show type grouping, and type 2 predominance is also common. ${ }^{7}$ The marked changes of the fibre types with a very few atrophic fibres found in the patient suggest that the denervating process would be chronic and be well compensated by re-innervation. Possible changes in permeability of the muscle membrane, brought by denervation and re-innervation, might cause leakage of $\mathrm{CK}$, resulting in high blood levels. His asymptomatic father also showed high CK levels and neurogenic changes in EMG, although there was no hypertrophy of the calves. This suggests that both the patient and his father were affected with the same disease, benign familial spinal muscle atrophy, inherited as an autosomal dominant trait.

An asymptomatic case of SMA with calf hypertrophy and high CK levels has not been described. Autosomal dominant spinal muscle atrophy is a relatively uncommon disease. The reported cases of juvenile proximal spinal muscle atrophy inherited as an autosomal dominant trait with complet penetrance $568-10$ generally showed benign course with slow or almost nळ progression in comparison with recessive spinal muscle atrophy, and some of the cases were described to present with hypertroph of the calves ${ }^{2}$ and elevated serum $\mathrm{CK}$ activity. ${ }^{25} 6^{6}$ D'Alessandro et $a l^{4}$ reported a fathew and son having benign spinal muscle atroe phy with muscle cramps, hypertrophy of the calves, and no loss of muscle strength. If their familia cases with autosomal dominant: inheritance, deep reflexes were diminisheof and serum CK levels were normal.

The patient may be completely asympe tomatic throughout his life because his father who is probably affected with the same disease remains asymptomatic in the seventh decade. A possibility cannot be ruled out that muscle weakness may appear later. The clinical spectrum must be furtheP investigated in such spinal muscle atrophy variants as our cases. This spinal muscles atrophy variant would be often clinicall. undetected because the patients have no complaints. Investigations of these asymptomatic cases are important for elucidating mechanisms of the onset and of the disegsen progression in motor neuron disease. The authors acknowledge the technical a $\frac{2}{8}$ is tance of Mrs Arai.

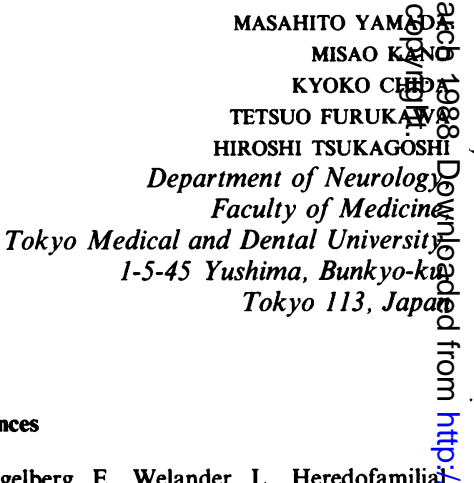

Kugelberg E, Welander L. Heredofamiliă juvenile muscular atrophy simulating mus. cular dystrophy. Arch Neruol Psychiaf 1956;75:500-9.

2 Tsukagoshi H, Sugita H, Furukawa T, Tsubak T, Ono E. Kugelberg-Welander syndrom with dominant inheritance. Arch Neuro 1966;14:378-81

3 Bouwsma G, Van Wijngaarden GK. Spinaゆ muscular atrophy and hypertrophy of the calves. J Neurol Sci 1980;44:275-9.

4 D'Alessandro R, Montagna P, Govoni E드. Pazzaglia P. Benign familial spinal muscula atrophy with hypertrophy of the calves. $\operatorname{Arck} \Omega$

Neurol 1982;39:657-60.
5 Zellweger H, Simpson J, McCormick WF Ionasescu V. Spinal muscular atrophy witho 
autosomal dominant inheritance. Report of a new kindred. Neurology 1972;22:957-63.

6 Sunohara N, Takada K, Ishihara T, Satoyoshi E. Juvenile onset proximal spinal muscular atrophy with autosomal dominant inheritance. Clin Neurol (Tokyo) 1980;20:547-54

7 Dubowitz V. Muscle Biopsy: A Practical Approach. 2nd ed. London: Bailliere Tindall, 1985.

8 Magee KR, DeJong RN. Neurogenic Muscular atrophy simulating muscular dystrophy. Arch Neurol 1960;2:677-82.

9 Garvie JM, Woolf AL. Kugelberg-Welander syndrome (hereditary proximal spinal muscular atrophy). Br med $J$ 1966;1:1458-61.

10 Armstrong RM, Fogelson MH, Silberberg DH. Familial proximal spinal muscular atrophy. Arch Neurol 1966:14:208-12.

Accepted 12 October 1987

\section{Reversible ataxia following chronic exposure to paradichlorobenzene}

- Sir: Paradichlorobenzene (PDCB) is mainly used as a moth repellant, mildew control agent and as a space deodorant. In spite of the broad spectrum of uses there are few reports of oral, inhalation or skin contact toxicity of this compound to man. Cataract formation, hepatic damage, ${ }^{1}$ anaemia, ${ }^{23}$ thrombocytopenia and lymphocytopenia ${ }^{4}$ have been reported following exposure to PDCB. However, no toxicity to the central nervous system (CNS) had been reported. We report a patient who developed cerebellar ataxia and speech disturbance following 6 years' exposure to PDCB.

A 25 year old woman was admitted to our hospital complaining of gait disturbance and dysarthria. She had been diagnosed as suffering from neurosis for 6 years. When - she was 19 years old (1980) the unusual idea that there were many tics in her room occurred to her and she learned to use moth balls of PDCB in her bedclothes and pillow as well as in her wardrobes. Moreover she began to grind them into powder and scattered it everywhere in her room. She put on her underwear which had been kept in bags filled with PDCB powder. Her mother says that there had been a strong odour of PDCB in her room for the last few years. In January 1986, clumsiness of both hands became apparent. She could not use chopsticks. She also found difficulty in gait and speech. Gradually her signs and symptoms became worse and she could not stand without support after 2 months. There was no fever. She was admitted to a hospital where neurological examination revealed severe cerebellar ataxia, dysarthria, moderate weakness of all limbs and hyporeflexia. Computed tomography of the brain, four vessel angiography and CSF findings were normal. Her symptoms and signs gradually improved after admission. A month later she was admitted to our hospital for further evaluation.

She was alert and well-nourished; neurological examination revealed moderate limb and truncal ataxia, dysarthria, hyporeflexia, hypotonus and mild proximal weakness of the four limbs. Blood pressure was $120 / 80 \mathrm{mmHg}$ with normal cardiac rhythm and there was no orthostatic hypotension. Normal laboratory data included blood count, electrolytes, liver and kidney function, serum creatine kinase, fast blood glucose, lactate, pyruvate, serologic reaction for syphilis and SLE, and thyroid function. Serum antibody titres to herpes, mumps, ECHO, varicella, Coxackie, polio, influenza, mycoplasma and EB virus were within normal limits. CSF findings were normal and oligoclonal bands were not detected. CSF culture and stains for bacteria including anti-fast bacilli and fungi were negative. Magnetic resonance imaging of the brain was normal. EEG, nerve conduction velocity, visual evoked potentials, blink reflexes, somatosensory evoked potentials to median nerve and posterior tibial nerve stimulation were also normal. Brainstem auditory evoked potentials (BAEPs) showed a marked delay of waves III, IV and V and elongation of II-V interval while latencies of waves I and II and I-II interval were normal (fig A). Her symptoms gradually improved and became minimal in 6 months after onset. BAEPs re-examined 8 months later were normal (fig B).

PDCB is a white crystalline compound which has been used for several decades as a repellent, space deodorant and fungicide (mildew-control agent). However, toxicity to the CNS of this substance had not been reported. In our case, continuous long-term exposure for 6 years is characteristic, and inhalation and local contact are considered to be the predominant routes of exposure. In experimental animals subjected to PDCB, intense eye and nose irritation, tremors and twitches of the extremities, a "mark time" reflex, a loss of the righting reflex, a definite nystagmus, rapid but laboured respiration, reversible granulocytopenia, kidney and lung injury, and some deaths have been reported. ${ }^{56}$ Hollingsworth et al also observed marked tremors, weakness, loss of weight, eye irritation, unkempt appearance,

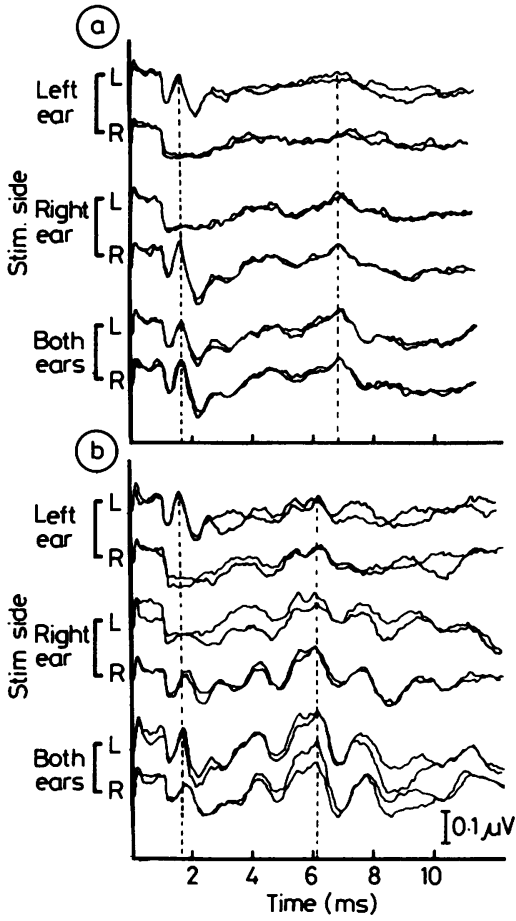

Fig (a) BAEPs 3 months after the onset. Marked delay of waves III, IV, and $V$, and elongation of II-V interval are shown. (b) BAEPs 8 months after the onset. $B A E P s$ are normal.

and unconsciousness in rats, guinea pigs, and rabbits subjected to repeated 8-hour exposures, 5 days a week, to the high concentration of PDCB vapour. Two of the rabbits survived 62 exposures and recovered completely. ${ }^{1}$ These facts suggest toxicity of PDCB to the CNS, although the result of microscopic examination of the CNS of exposed animals was not mentioned Reversibility of symptoms seen in the experimental models reported by $\mathrm{Hol}$ lingsworth et al and our case may be partially attributed to rapid elimination of this substance. ${ }^{7}$ Acute cerebellar ataxia and brainstem encephalitis should be ruled out in our case. However, there was no preceding infection and no fever, speed of worsening was rather slow, and symptoms improved after cessation of exposure Abnormalities of BAEPs have not been reported in acute cerebellar ataxia. Therefore long-term exposure of PDCB may be 